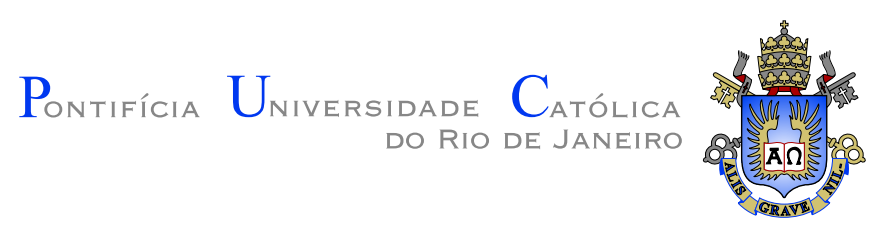

Andréa Cynthia dos Santos

\title{
Modelos e Algoritmos para o Problema da Árvore Geradora de Custo Mínimo com Restrição de Diâmetro
}

Tese apresentada ao Programa de Pós-graduação em Informática do Departamento de Informática da PUC-Rio como requisito parcial para obtenção do título de Doutor em Informática

Orientador : Prof. Celso da Cruz Carneiro Ribeiro

Co-Orientador: Prof. Abilio Pereira Lucena Filho 


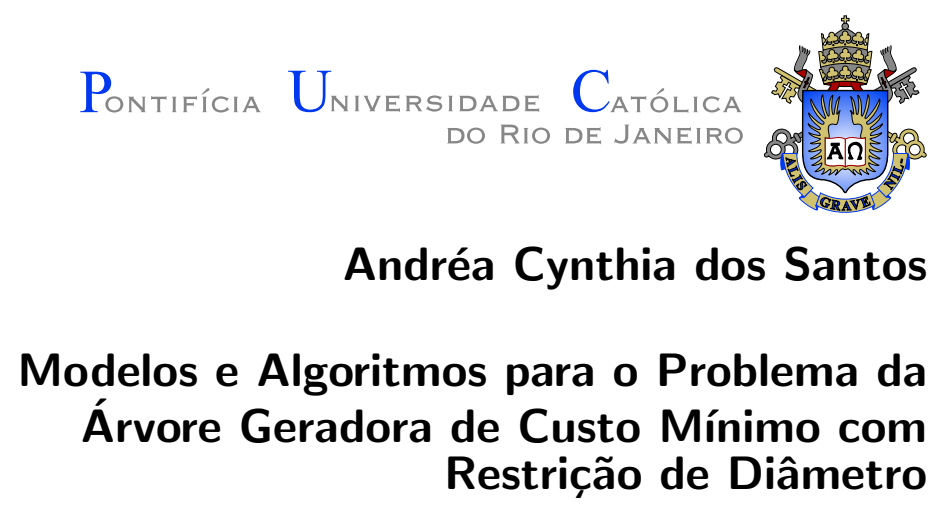

Tese apresentada ao Programa de Pós-graduação em Informática do Departamento de Informática do Centro Técnico Científico da PUC-Rio como requisito parcial para obtenção do título de Doutor em Informática. Aprovada pela Comissão Examinadora abaixo assinada.

\section{Prof. Celso da Cruz Carneiro Ribeiro Orientador Departamento de Informática - PUC-Rio}

Prof. Abilio Pereira Lucena Filho Co-Orientador Departamento de Administração - UFRJ

Prof. Noemi de la Rocque Rodriguez Presidente Departamento de Informática - PUC-Rio Prof. Nelson Maculan Filho Programa de Engenharia de Sistemas e Computação - UFRJ

Prof. Philippe Yves Paul Michelon Departamento de Informática - Universidade de Avignon, França

Prof. Simone de Lima Martins Departamento de Ciência da Computação - UFF

Prof. Luiz Satoru Ochi Departamento de Ciência da Computação - UFF

Prof. José Eugênio Leal Coordenador Setorial do Centro Técnico Científico - PUC-Rio 
Todos os direitos reservados. É proibida a reprodução total ou parcial do trabalho sem autorização da universidade, do autor e do orientador.

\section{Andréa Cynthia dos Santos}

Graduou-se em Ciência da Computação na Universidade Federal do Rio Grande do Norte (Natal, Brasil). Desenvolveu o Sistema de Automação do Mestrado (SAM) como projeto final do Curso de Ciência da Computação. Obteve o título de mestre em Informática na Universidade Federal do Rio Grande do Norte (Natal, Brasil). Desenvolveu o Sistema de Gerenciamento de Unidades Móveis de Pistoneio (SGUMP) durante o Mestrado junto do seu orientador, da Petrobras e da Agência Nacional de Petróleo (ANP). Participou do Programa de Doutorado no País com Estágio no Exterior (PDEE), permanecendo seis meses do doutorado no Laboratório de Informática da Universidade de Avignon (França).

Ficha Catalográfica

Santos, Andréa Cynthia dos

Modelos e Algoritmos para o Problema da Árvore Geradora de Custo Mínimo com Restrição de Diâmetro / Andréa Cynthia dos Santos; orientador: Celso da Cruz Carneiro Ribeiro; co-orientador: Abilio Pereira Lucena Filho. - Rio de Janeiro : PUC-Rio, Departamento de Informática, 2006.

133 f: il. ; $30 \mathrm{~cm}$

1. Tese (doutorado) - Pontifícia Universidade Católica do Rio de Janeiro, Departamento de Informática.

Inclui referências bibliográficas.

1. Informática - Tese. 2. Árvore Geradora de Custo Mínimo. 3. Problemas NP-difíceis. 4. Projeto de Redes. 5. Relaxação Lagrangeana. 6. Metaheurísticas. 7. Heurísticas Híbridas. I. Ribeiro, Celso da Cruz Carneiro. II. Lucena Filho, Abilio Pereira. III. Pontifícia Universidade Católica do Rio de Janeiro. Departamento de Informática. IV. Título. 


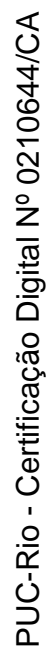

Dedico este trabalho, com amor e carinho, a minha mãe Rita Santos. 


\section{Agradecimentos}

Aos meus orientadores Celso Ribeiro e Abilio Lucena pela oportunidade que me deram de trabalhar com os dois, por terem conduzido este trabalho incentivando e advertindo quando necessário, pela disponibilidade em compartilhar suas experiências, por abrirem novas portas do conhecimento para mim e pelo apoio nos momentos difíceis.

A minha mãe Rita Santos pelo apoio incondicional e por suas palavras diárias de encorajamento, amizade e carinho.

Ao estimado Christophe Duhamel por sua compreensão, cumplicidade e carinho. Particularmente, por sua contribuição no desenvolvimento do visualizador de instâncias.

À CAPES pelo apoio financeiro que viabilizou o desenvolvimento deste trabalho de tese. Especialmente, pelo apoio financeiro e logístico durante o meu estágio na França.

Ao professor Philippe Michelon e sua equipe por terem me recebido tão bem no laboratório de Informática de Avignon durante o meu estágio na França.

À professora Noemi Rodriguez por me convidar para ministrar alguns cursos na Petrobras e pela bolsa parcial concedida após o término da bolsa CAPES.

Aos amigos Thiago Noronha, Sebastiàn Urrutia, Adriana Alvim e Carlos Eduardo Vieira pelos momentos de amizade e troca de conhecimentos. Também agradeço o apoio de outros amigos conquistados durante o doutorado.

Aos funcionários do suporte José Carlos, Anderson Oliveira, Luciana Almeida e ao estagiário Túlio Peixoto pela boa vontade em sanar os problemas do laboratório.

Aos funcionários Deborah Gonçalves da secretaria, Rosane Teles e Cosme Leal da biblioteca pela atenção e eficiência no atendimento das minhas solicitações.

Aos professores Luiz Fernando Seibel e Celso Ribeiro pela oportunidade de realizar um estágio de seis meses em bioinformática.

Ao professor Dario Aloise pela amizade e pelos incentivos dados para que eu seguisse uma carreira acadêmica. 


\section{Resumo}

Santos, Andréa Cynthia dos; Ribeiro, Celso da Cruz Carneiro; Lucena Filho, Abilio Pereira. Modelos e Algoritmos para o Problema da Árvore Geradora de Custo Mínimo com Restrição de Diâmetro. Rio de Janeiro, 2006. 133p. Tese de Doutorado - Departamento de Informática, Pontifícia Universidade Católica do Rio de Janeiro.

Nesta tese são propostos modelos e algoritmos aproximados para o Problema da Árvore Geradora de Custo Mínimo com Restrição de Diâmetro (AGMD). Este problema modela tipicamente aplicações em projetos de redes de computadores onde todos os vértices devem comunicar-se entre si a um custo mínimo, garantindo um certo nível de serviço. Os modelos propostos por Achuthan e Caccetta para o AGMD são reforçados através da introdução de restrições válidas. Uma relaxação lagrangeana é proposta para o modelo de multifluxo básico de Gouveia e Magnanti. Essa relaxação é utilizada para o desenvolvimento de heurísticas lagrangeanas. Adaptações são realizadas nas heurísticas construtivas propostas por Deo e Abdalla, e por Raidl e Julstrom. São propostas ainda quatro estratégias de busca local, uma heurística do tipo GRASP e outra híbrida. São obtidos limites superiores a menos de $2 \%$ do ótimo para as classes de instâncias usadas nos trabalhos de Gouveia e Magnanti, e de Santos, Lucena e Ribeiro. Além disto, obteve-se os melhores resultados conhecidos até o presente momento para 11 instâncias de grafos completos usadas por Raidl, Julstrom e Gruber.

\section{Palavras-chave}

Árvore Geradora de Custo Mínimo. Problemas NP-difíceis. Projeto de Redes. Relaxação Lagrangeana. Metaheurísticas. Heurísticas Híbridas. 


\section{Abstract}

Santos, Andréa Cynthia dos; Ribeiro, Celso da Cruz Carneiro; Lucena Filho, Abilio Pereira. Models and algorithms for the Diameter Constrained Minimum Spanning Tree Problem. Rio de Janeiro, 2006. 133p. PhD Thesis - Department of Informática, Pontifícia Universidade Católica do Rio de Janeiro.

In this work, models and approximation algorithms to solve the Diameter Constrained Minimum Spanning Tree Problem (AGMD) are proposed. This problem typically models network design applications where all vertices must communicate with each other at a minimum cost, while meeting a given quality requirement. The formulations proposed by Achuthan and Caccetta are strengthened with valid inequalities. A lagrangean relaxation is proposed for the multicommodity flow model developed by Gouveia and Magnanti. Adaptations are made in the constructive heuristics proposed by Deo and Abdalla and by Raidl and Julstrom. Four local search procedures, a GRASP algorithm and a hybrid heuristic are proposed. Upper bounds within $2 \%$ of the optimal solution values are obtained for the two classes of instances used by Gouveia and Magnanti and by Santos, Lucena and Ribeiro. Moreover, stronger upper bounds are reported for 11 instances in complete graphs used by Raidl, Julstrom and Gruber.

\section{Keywords}

Minimum Spanning Tree. NP-Hard Problems. Network Design. Lagrangean Relaxation. Metaheuristics. Hybrid Heuristics. 


\section{Sumário}

1 Introdução

2 O problema da árvore geradora de custo mínimo com restrição de diâmetro 14

$\begin{array}{lll}2.1 & \text { Problemas correlatos } & 15\end{array}$

$\begin{array}{lll}2.2 & \text { Revisão bibliográfica } & 15\end{array}$

$\begin{array}{lll}2.3 & \text { Modelos para AGMD } & 18\end{array}$

2.4 Heurísticas e metaheurísticas presentes na literatura 26

3 Extensões dos modelos matemáticos $\quad 36$

3.1 Uma formulação alternativa para o caso $D$ ímpar 36

3.2 Restrições válidas 38

$\begin{array}{lll}3.3 & \text { Lifting } & 38\end{array}$

3.4 Experimentos computacionais 40

3.5 Conclusões 46

4 Relaxação lagrangeana para AGMD $\quad 47$

4.1 Fundamentos básicos da técnica 47

4.2 Uma relaxação lagrangeana para o modelo multifluxo básico 51

4.3 Algoritmo de solução 54

4.4 Experimentos computacionais da relaxação lagrangeana 57

$\begin{array}{ll}4.5 \text { Conclusões } & 67\end{array}$

$\begin{array}{lll}5 & \text { Heurísticas construtivas para AGMD } & 68\end{array}$

$\begin{array}{lll}5.1 & \text { Heurísticas construtivas semi-gulosas } & 68\end{array}$

5.2 Variantes da heurística OTT 69

5.3 Variantes da heurística RGH

5.4 Experimentos computacionais $\quad 74$

5.5 Conclusões 88

6 Buscas locais e heurísticas baseadas em metaheurísticas para AGMD $\quad 89$

$\begin{array}{lll}6.1 & \text { Vizinhanças e buscas locais } & 89\end{array}$

6.2 Otimizações realizadas nas buscas locais $\quad 97$

6.3 Heurísticas baseadas em metaheurísticas $\quad 97$

6.4 Um algoritmo GRASP para AGMD 100

6.5 Um algoritmo híbrido para AGMD 102

$7 \quad$ Experimentos computacionais $\quad 108$

7.1 Experimentos computacionais com as heurísticas lagrangeanas 108

7.2 Experimentos computacionais com as heurísticas baseadas em metaheurísticas 111

$\begin{array}{ll}7.3 \text { Conclusões } & 124\end{array}$

8 Conclusões e trabalhos futuros $\quad 125$ 


\section{Lista de figuras}

1.1 Exemplo para o caso $D=2.11$

2.1 Casos polinomiais. 14

2.2 Inserção de um nó artificial no caso ímpar por Gouveia, Magnanti e Requejo [29]. 17

2.3 Inserção de um nó artificial no caso ímpar por Santos, Lucena e $\begin{array}{ll}\text { Ribeiro [60]. } & 17\end{array}$

2.4 Restrição válida para o modelo básico. 23

2.5 Exemplo de falsa violação de diâmetro. 26

3.1 Solução para AGMD caso ímpar com $D=3$.

4.1 Convergêngia obtida com $\tau_{\pi}=10$ e $\tau=500$. 62

4.2 Convergêngia obtida com $\tau_{\pi}=25$ e $\tau=500$.

6.1 Exemplo de migração para uma solução vizinha utilizando 1-opt. 90

6.2 Exemplo de migração para uma solução vizinha utilizando 2-opt. 93

6.3 Exemplo de soluções vizinhas na vizinhança adoção de sub-árvore. 95

6.4 Exemplo de movimento da vizinhança substituição de caminho. 96

6.5 Exemplo da perturbação DCV. 103

6.6 Exemplo da perturbação SAR. 104

7.1 Exemplos de uma solução para o caso $D$ par. 117

7.2 Exemplos de uma solução para o caso $D$ ímpar. 118

7.3 Distribuição de probabilidade no tempo (Grupo 2 - alvos 131 e 130).120

7.4 Distribuição de probabilidade no tempo (Grupo 2 - alvos 129 e 128).121

7.5 Distribuição de probabilidade no tempo (Grupo 3 - alvos 7,06 e 7,12). 


\section{Lista de tabelas}

2.1 Resultados apresentados na literatura das heurísticas baseadas em metaheurísticas.

3.1 Resultados para as instâncias do Grupo 1 (caso $D$ ímpar). 43

3.2 Resultados para as instâncias do Grupo 1 (caso $D$ par). 44

3.3 Resultados para as instâncias do Grupo 2 (caso $D$ ímpar). 45

4.1 Primeiro experimento de calibração. 61

4.2 Segundo experimento de calibração (primeira execução). 63

4.3 Segundo experimento de calibração (segunda execução). 64

4.4 Limites inferiores para as instâncias do Grupo 1.

5.1 Resumo dos resultados obtidos para as instâncias do Grupo $1 . \quad 76$

5.2 Resumo dos resultados obtidos para as instâncias do Grupo 2.

5.3 Resumo dos resultados obtidos para as instâncias do Grupo 3. 76

5.4 Resultados das heurísticas para as instâncias do Grupo 1.

5.5 Continuação dos resultados das heurísticas para as instâncias do Grupo $1 . \quad 80$

5.6 Resultados das heurísticas para as instâncias do Grupo 2.

5.7 Continuação dos resultados das heurísticas para as instâncias do Grupo 2.

5.8 Resultados das heurísticas para as instâncias do Grupo 3. 82

5.9 Continuação dos resultados das heurísticas para as instâncias do Grupo 3.

5.10 Tempos de processamento para as instâncias do Grupo 3. 84

5.11 Resultados do segundo experimento para as instâncias do Grupo 3. 86

5.12 Continuação dos resultados do segundo experimento para as instâncias do Grupo 3.

7.1 Resultados das heurísticas lagrangeanas para as instâncias do Grupo 1.

7.2 Resultados das heurísticas lagrangeanas para as instâncias do Grupo 2.

7.3 Resultados das heurísticas lagrangeanas para algumas instâncias do Grupo 3.

7.4 Resultados dos testes do filtro utilizado na perturbação. 112

7.5 Resultados do segundo experimento para o Grupo 1.

7.6 Resultados do segundo experimento para o Grupo 2. 115

7.7 Resultados do segundo experimento para o Grupo 3. 116

7.8 Resultados dos testes com as perturbações para o Grupo 2. 123

7.9 Resultados dos testes com as perturbações para o Grupo 3. 123 